Research Article

\title{
General Complex-Valued Grouping Functions
}

\author{
Ying Chen, ${ }^{1}$ Lvqing $\mathrm{Bi}^{2}{ }^{2} \mathrm{Bo} \mathrm{Hu}^{3}$ and Songsong Dai $\mathbb{D}^{1}$ \\ ${ }^{1}$ School of Electronics and Information Engineering, Taizhou University, Taizhou 318000, China \\ ${ }^{2}$ School of Electronics and Communication Engineering, \\ Guangxi Colleges and Universities Key Laboratory of Complex System Optimization and Big Data Processing, \\ Yulin Normal University, Yulin 537000, China \\ ${ }^{3}$ School of Big Data and Computer Science, Guizhou Normal University, Guiyang 550025, China
}

Correspondence should be addressed to Songsong Dai; ssdai@stu.xmu.edu.cn

Received 8 April 2021; Revised 26 May 2021; Accepted 7 August 2021; Published 14 August 2021

Academic Editor: Feng Feng

Copyright (๑) 2021 Ying Chen et al. This is an open access article distributed under the Creative Commons Attribution License, which permits unrestricted use, distribution, and reproduction in any medium, provided the original work is properly cited.

Grouping function is a special kind of aggregation function which measures the amount of evidence in favor of either of the two choices. Recently, complex fuzzy sets have been successfully used in many fields. This paper extends the concept of grouping functions to the complex-valued setting. We introduce the concepts of complex-valued grouping, complex-valued 0-grouping, complex-valued 1-grouping, and general complex-valued grouping functions. We present some interesting results and construction methods of general complex-valued grouping functions.

\section{Introduction}

In 2012, Bustince et al. [1] introduced the concept of grouping function as a special type of aggregation functions [2]. A grouping function measures the amount of evidence in favor of either of two choices in decision making. It plays an important role in many aspects of applications such as image processing $[1,3]$, classification $[4,5]$, and decision making $[6,7]$.

In this paper, we consider complex-valued grouping function, which is both a generation of real-valued grouping function and a special kind of complex fuzzy aggregation operator. In the literature, one can find many works on realvalued grouping functions. The concepts of general grouping functions [8] and interval-valued grouping functions $[9,10]$ have been proposed. Some properties incluing migrativity, homogeneity, idempotency, and distributivity of grouping functions have been studied [1, 11-14]. The multiplicative generators [15] and additive generators [16] of grouping functions have been investigated. (G, N)-implications [17] and QL-implications [18] derived from grouping functions have been constructed.

Meanwhile, we can also find several works on complex fuzzy aggregation operators and related concepts. In 2002,
Ramot et al. [19, 20] introduced the concepts of complex fuzzy sets and complex fuzzy aggregation operators, which have been successfully used in signal processing [20-22], time series forecasting [23-25], and decision making $[26,27]$. Distance and entropy measures of complex fuzzy sets and its extensions have been proposed [28-30]. Moreover, some new concepts such as orthogonality and rotational invariance have been presented for complex fuzzy sets and complex fuzzy aggregation operators [31-33].

Recently, Chen et al. [34] introduced the concept of complex-valued overlap function, which measures the overlapping degree between two objects with complexvalued information. In order to measure the amount of evidence in favor of either of two choices with complexvalued information, this paper extends traditional realvalued grouping functions to complex-valued grouping functions. As mentioned in [34], some features of complex fuzzy sets can lead to special properties for complex-valued overlap functions.

Both real-valued grouping functions and complex fuzzy aggregation operators have gained a rapid development both in application and theory. However, as far as we know, nowadays, there are no corresponding discussions to propose the complex-valued grouping functions. Therefore, in 
this paper, we introduce the concepts of complex-valued grouping functions. This paper is organized as follows. In Section 2, we recall the concepts of grouping functions. In Section 3, we introduce complex-valued grouping functions and their properties. In Section 4, we introduce construction methods of general complex-valued grouping functions. Conclusions are given in Section 5.

\section{Preliminaries}

In this section, we recall the concepts of bivariate grouping functions and n-dimensional grouping functions $[1,8,12,16]$.

\subsection{Overlap Functions}

Definition 1 (see [1]). A mapping $G:[0,1]^{2} \longrightarrow[0,1]$ is a grouping function if it is symmetric, nondecreasing, continuous, and has the following properties:

(G1) $G(a, b)=0$ if and only if $a=b=0$

(G2) $G(a, b)=1$ if and only if $a=1$ or $b=1$

As introduced in [16], a mapping $G:[0,1]^{2} \longrightarrow[0,1]$ is a 0 -grouping function if we replace the property $(\mathrm{G} 1)$ by the following:

(G1') $a=b=0 \Longrightarrow G(a, b)=0$ without changing others.

Similarly, a mapping $G:[0,1]^{2} \longrightarrow[0,1]$ is a 1 -grouping function if we replace the property (G2) by the following:

(G2') $a=1$ or $b=1 \Longrightarrow O(a, b)=1$ without changing others.

Definition 2 (see [12]). An $n$-ary mapping $G_{n}:[0,1]^{n} \longrightarrow[0,1]$ is an $n$-dimensional grouping function if it is commutative, nondecreasing, continuous, and has the following properties:

$\left(G_{n} 1\right) G_{n}\left(a_{1}, \ldots, a_{n}\right)=0$ if and only if $a_{i}=0$ for all $i=1,2, \ldots, n$

$\left(G_{n} 2\right) G_{n}\left(a_{1}, \ldots, a_{n}\right)=1$ if and only if there exists $i \in\{1,2, \ldots, n\}$ such that $a_{i}=1$

Analogously, an $n$-ary mapping $G_{n}:[0,1]^{n} \longrightarrow[0,1]$ is an $n$-dimensional 0 -grouping function if we replace the property $\left(G_{n} 1\right)$ by the following:

$\left(G_{n} 1^{\prime}\right)$ If $a_{i}=0$ for all $i=1,2, \ldots, n$, then $G_{n}\left(a_{1}, \ldots, a_{n}\right)=0$ without changing others.

An $n$-ary mapping $G_{n}:[0,1]^{n} \longrightarrow[0,1]$ is an $n$-dimensional 1-grouping function if we replace the property $\left(G_{n} 2\right)$ by the following:

$\left(G_{n} 2^{\prime}\right)$ If there exists $i \in\{1,2, \ldots, n\}$ such that $a_{i}=1$, then $G_{n}\left(a_{1}, \ldots, a_{n}\right)=1$ without changing others.

Based on the concepts of $n$-dimensional 0 -grouping and 1-grouping functions, the general grouping functions is defined as follows.
Definition 3 (see [8]). A mapping $G_{n}:[0,1]^{n} \longrightarrow[0,1]$ is an $n$-dimensional general grouping function if it is commutative, nondecreasing, continuous, and has the following properties:

$\left(G g_{n} 1\right)$ If $\sum_{i=1}^{n} a_{i}=0$ then $G_{n}\left(a_{1}, \ldots, a_{n}\right)=0$

$\left(G g_{n} 2\right)$ If there exists $i \in\{1,2, \ldots, n\}$ such that $a_{i}=1$, then $G_{n}\left(a_{1}, \ldots, a_{n}\right)=1$

\section{3. $\boldsymbol{N}$-Dimensional Complex-Valued Grouping Functions}

Let $D=\{\alpha \in \mathbb{C}|| \alpha \mid \leq 1\}$, each $a \in D$ is of the form $r_{a} e^{j \theta_{a}}$, where $j=\sqrt{-1}$, the amplitude term $r_{a} \in \mathbb{R}$ and the phase term $\theta_{a} \in[0,2 \pi)$.

We define $n$-dimensional complex-valued grouping functions.

Definition 4. An $n$-ary mapping $C g_{n}: D^{n} \longrightarrow D$ is an $n$-dimensional complex-valued grouping function if it is commutative, continuous, and has the following properties:

$\left(C g_{n} 1\right) C g_{n}\left(a_{1}, \ldots, a_{n}\right)=0$ if and only if $a_{i}=0$ for all $i=1,2, \ldots, n$

$\left(C g_{n} 2\right) C g_{n}\left(a_{1}, \ldots, a_{n}\right)=1$ if and only if there exists $i \in\{1,2, \ldots, n\}$ such that $a_{i}=1$

$\left(C g_{n} 3\right) C g_{n}$ is amplitude monotonic in the first variate: $\left|C g_{n}\left(a, a_{2}, \ldots, a_{n}\right)\right| \leq\left|C g_{n}\left(b, a_{2}, \ldots, a_{n}\right)\right|$ when $|a| \leq|b|$

Since $\mathrm{Cg}_{n}$ is commutative, it is also amplitude monotonic in any other variate based on the third property $\left(\mathrm{Cg}_{n} 3\right)$. Obviously, these properties are analogous to those of Definition 1 . When the domain is limited to $[0,1]$, it reduces to an $n$-dimensional real-valued grouping function of Definition 2.

Example 1. Nevertheless, there exists mapping such that is a grouping function in the domain $[0,1]$ but not a complexvalued grouping function. The function given as follows:

$$
f(a, b)=1-(1-a)(1-b)
$$

is a grouping function but not a complex-valued grouping function; for example, $f(0.5,-1)=0$, then $\left(C g_{n} 1\right)$ does not hold.

Traditional real-valued grouping functions are the dual notion of overlap functions. $f(a, b)$ is an overlap function on $[0,1]$, and $g(a, b)=1-f(1-a, 1-b)$ is a grouping function. However, this is not true for complex-valued overlap functions. For example, $f(a, b)=a b$ is a complexvalued overlap function on $D$ in [34], but $g(a, b)=1-(1-$ a) $(1-b)$ is not a complex-valued grouping function.

Similarly, we introduce some types of grouping functions, such as n-dimensional complex-valued 0-overlap and 1-overlap grouping functions.

An $n$-ary mapping $C g_{n}: D^{n} \longrightarrow D$ is an n-dimensional complex-valued 0 -grouping function if we replace the property $\left(C g_{n} 1\right)$ by the following:

$\left(C g_{n} 1^{\prime}\right)$ If $a_{i}=0$ for all $i=1,2, \ldots, n$, then $C g_{n}\left(a_{1}, \ldots, a_{n}\right)=0$ without changing others. 
An $n$-ary mapping $C g_{n}: D^{n} \longrightarrow D$ is an n-dimensional complex-valued 1-grouping function if we replace the property $\left(C g_{n} 2\right)$ by the following:

$\left(C g_{n} 2^{\prime}\right)$ If there exists $i \in\{1,2, \ldots, n\}$ such that $a_{i}=1$ then $C g_{n}\left(a_{1}, \ldots, a_{n}\right)=1$ without changing others.

Based on these concepts, we define the concept of $n$ dimensional general complex-valued grouping functions.

Definition 5. An $n$-ary mapping $G C g_{n}: D^{n} \longrightarrow D$ is an $n$-dimensional general complex-valued grouping function if it is commutative, continuous, and has the following properties:

$\left(G C g_{n} 1\right)$ If $a_{i}=0$ for all $i=1,2, \ldots, n$, then $\operatorname{GCg}_{n}\left(a_{1}, \ldots, a_{n}\right)=0$

$\left(G C g_{n} 2\right)$ If there exists $i \in\{1,2, \ldots, n\}$ such that $a_{i}=1$ then $G C g_{n}\left(a_{1}, \ldots, a_{n}\right)=1$

$\left(G C g_{n} 3\right) G C g_{n}$ is amplitude monotonic in the first variate: $\quad\left|G C g_{n}\left(a, a_{2}, \ldots, a_{n}\right)\right| \leq\left|G C g_{n}\left(b, a_{2}, \ldots, a_{n}\right)\right|$ when $|a| \leq|b|$

The relations between $n$-dimensional complex-valued grouping functions, complex-valued 0-grouping functions, complex-valued 1-grouping functions, and general complex-valued grouping functions are given as follows.

Proposition 1. Let $g: D^{n} \longrightarrow D$ be an $n$-ary mapping, then

(1) If it is an n-dimensional complex-valued grouping function, then it is an n-dimensional complex-valued 0 -grouping and 1-grouping function

(2) If it is an n-dimensional complex-valued 0-grouping (or 1-grouping) function, then it is also a general complex-valued grouping function

This relation between complex-valued grouping functions is similar to that between interval-valued overlap functions [9].

Now, we give several examples to demonstrate their relations of complex-valued grouping functions.

Example 2. The binary function $g: D^{2} \longrightarrow D$ given by

$$
g(a, b)=\max (|a|,|b|) \cdot e^{\theta_{a} \wedge \theta_{b}},
$$

is a complex-valued overlap function.

Example 3. The binary function $f: D^{2} \longrightarrow D$ given by

$$
f(a, b)=|a| \vee|b|,
$$

is a general complex-valued grouping function and complex-valued 1-grouping function, but neither a complexvalued grouping function nor a complex-valued 0-grouping function; for example, $f(-1,-1)=1$, then the property $\left(\mathrm{Cg}_{n} 2\right)$ does not hold.

Example 4. The binary function $h: D^{2} \longrightarrow D$ given by

$$
h(a, b)= \begin{cases}1, & a=1 \text { or } b=1, \\ 0, & \text { otherwise, }\end{cases}
$$

is a general complex-valued grouping function and complex-valued 0 -grouping function. However, $h$ is not a complex-valued 1-grouping function; for example, $f(0.5,0.5)=0$, then the property $\left(C g_{n} 1\right)$ does not hold.

Negative operation (-) is closed on $D$ but not closed on $[0,1]$. Then, we have following property only for complexvalued function on $D$.

Definition 6. The $n$-ary function $g: D^{n} \longrightarrow D$ is symmetric with respect to the point 0 , if

$$
g\left(a_{1}, \ldots, a_{n}\right)=g\left(-a_{1},-a_{2}, \ldots,-a_{n}\right),
$$

holds for any $a_{1}, \ldots, a_{n} \in D$.

Example 5. The $n$-ary function $g: D^{n} \longrightarrow D$ given by

$$
g\left(a_{1}, \ldots, a_{n}\right)=\min \left(n-\sum_{i=1}^{n}\left(1-\left|a_{i}\right|\right), 1\right) \cdot e^{\theta_{a_{1}} \wedge \cdots \wedge \theta_{a_{n}}},
$$

is a complex-valued grouping function, which is symmetric with respect to the point 0 .

\section{Construction of General Complex-Valued Overlap Functions}

Proposition 2. If an n-ary mapping $\varphi: D^{n} \longrightarrow D$ is $n$-dimensional complex-valued grouping (0-grouping, 1-grouping, or general grouping) function which is expressed as

$$
\varphi\left(a_{1}, \ldots, a_{n}\right)=\phi\left(r_{a_{1}}, r_{a_{2}}, \ldots, r_{a_{n}}\right) e^{j \psi\left(\theta_{a_{1}}, \theta_{a_{2}}, \ldots, \theta_{a_{n}}\right)},
$$

then the function $\phi$ is an $n$-dimensional grouping (0-grouping, 1 -grouping, or general grouping) function on $[0,1]$.

Theorem 1. If the n-ary function $\phi:[0,1]^{n} \longrightarrow[0,1]$ is an $n$-dimensional 1-grouping function, the function $\psi:[0,2 \pi)^{n} \longrightarrow[0,2 \pi)$ satisfies the following properties:

(i) $\psi$ is commutative

(ii) There exists $i \in\{1,2, \ldots, n\}$ such that $\theta_{a_{i}}=0$ if and only if $\psi\left(\theta_{a_{1}}, \ldots, \theta_{a_{n}}\right)=0$

(iii) $\psi$ is continuous

Then, the function $\varphi: D^{n} \longrightarrow D$ defined by equation (7) is an n-dimensional complex-valued 1-grouping function.

Proof. It is immediate that $\varphi$ is commutative, amplitude monotonic, and continuous. Now, we prove the properties $\left(\mathrm{Cg}_{n} 1\right)$ and $\left(\mathrm{Cg}_{\mathrm{n}} 2^{\prime}\right)$.

$\left(C g_{n} 1\right): \quad(\Rightarrow)$ If $\varphi\left(a_{1}, \ldots, a_{n}\right)=\phi\left(r_{a_{1}}, r_{a_{2}}, \ldots, r_{a_{n}}\right)$ $e^{j \psi\left(\theta_{a_{1}}, \theta_{a_{2}}, \ldots, \theta_{a_{n}}\right)}=0$, then $\phi\left(r_{a_{1}}, r_{a_{2}}, \ldots, r_{a_{n}}\right)=0$. Then, $r_{a_{i}}=0$ for all $i=1,2, \ldots, n$ since $\phi$ is a 1-grouping function. Then, $a_{i}=0$ for all $i=1,2, \ldots, n$. 
$\left(C g_{n} 1\right):(\Leftarrow)$ If $a_{i}=0$ for all $i=1,2, \ldots, n$, this means $r_{a_{i}}=0$ for all $i=1,2, \ldots, n$, and then $\phi\left(r_{a_{1}}, r_{a_{2}}, \ldots, r_{a_{n}}\right)=0$ since $\phi$ is a 1-grouping function. Then, $\varphi\left(a_{1}, \ldots, a_{n}\right)=\phi\left(r_{a_{1}}, r_{a_{2}}, \ldots, r_{a_{n}}\right) e^{j \psi} \quad\left(\theta_{a_{1}}, \theta_{a_{2}}\right.$, $\left.\ldots, \theta_{a_{n}}\right)=0 \cdot e^{j \psi\left(\theta_{a_{1}}, \theta_{a_{2}}, \ldots, \theta_{a_{n}}\right)}=0$.

$\left(\mathrm{Cg}_{\mathrm{n}} 2^{\prime}\right):(\Leftarrow)$ If there exists $i \in\{1,2, \ldots, n\}$ such that $a_{i}=1$, i.e., $r_{a_{i}}=1$ and $\theta_{a_{i}}=0$, then $\phi\left(r_{a_{1}}, r_{a_{2}}, \ldots, r_{a_{n}}\right)=$ 1 since $\phi$ is a 1-grouping function, and $\psi\left(\theta_{a_{1}}, \theta_{a_{2}}, \ldots, \theta_{a_{n}}\right)=0$ since $\psi$ satisfies (ii). Then, $\varphi\left(a_{1}, \ldots, a_{n}\right)=\phi\left(r_{a_{1}}, r_{a_{2}}, \ldots, r_{a_{n}}\right) e^{j \psi\left(\theta_{a_{1}}, \theta_{a_{2}}, \ldots, \theta_{a_{n}}\right)}=1$. $e^{j 0}=1$.

Corollary 1. If the $n$-ary function $\phi:[0,1]^{n} \longrightarrow[0,1]$ is an $n$-dimensional general grouping function, the function $\psi:[0,2 \pi)^{n} \longrightarrow[0,2 \pi)$ satisfies the following properties:

(i) $\psi$ is commutative

(ii) There exists $i \in\{1,2, \ldots, n\}$ such that $\theta_{a_{i}}=0$ if and only if $\psi\left(\theta_{a_{1}}, \ldots, \theta_{a_{n}}\right)=0$

(iii) $\psi$ is continuous

Then, the function $\varphi: D^{n} \longrightarrow D$ defined by equation (7) is an $n$-dimensional general complex-valued grouping function.

However, this method cannot obtain complex-valued grouping function. For example, we get $r_{a_{i}}=1$ from $\phi$ is a grouping function and $\theta_{a_{k}}=0$ from property (ii), but $i \neq k$; thus, we cannot get $a_{i}=1$. So, we consider the construction of general complex-valued overlap functions. If the $n$-dimensional complex-valued function $\varphi: D^{n} \longrightarrow D$ is defined by equation (7), then we can easily see that it is a key step to construct the function $\psi:[0,2 \pi)^{n} \longrightarrow[0,2 \pi)$, which satisfies the condition (ii) of Corollary 1.

Now, we give some examples of bivariate functions $\psi:[0,2 \pi)^{2} \longrightarrow[0,2 \pi)$ satisfying the condition (ii) of Corollary 1 .

Example 6. The $n$-ary function $\psi_{1}:[0,2 \pi)^{n} \longrightarrow[0,2 \pi)$ given by

$$
\psi_{1}\left(\theta_{1}, \theta_{2}, \ldots, \theta_{n}\right)=\frac{\theta_{1}}{2 \pi} \times \frac{\theta_{2}}{2 \pi} \times \cdots \times \frac{\theta_{n}}{2 \pi},
$$

satisfies the condition (ii) of Corollary 1.

The $n$-ary function $\psi_{2}:[0,2 \pi)^{n} \longrightarrow[0,2 \pi)$ given by

$$
\psi_{2}\left(\theta_{1}, \theta_{2}, \ldots, \theta_{n}\right)=\theta_{1} \wedge \theta_{2} \wedge \cdots \wedge \theta_{n},
$$

satisfies the condition (ii) of Corollary 1.

Based on these functions, we give the general complexvalued grouping functions.

The function $\varphi_{1}: D^{n} \longrightarrow D$ given by

$$
\varphi_{1}\left(a_{1}, \ldots, a_{n}\right)=\left(r_{a_{1}} \vee r_{a_{2}} \vee \cdots \vee r_{a_{n}}\right) \cdot e^{j \psi_{1}\left(\theta_{1}, \theta_{2}, \ldots, \theta_{n}\right)} \text {, }
$$

is a general complex-valued grouping function.

The function $\varphi_{2}: D^{n} \longrightarrow D$ given by

$$
\varphi_{2}\left(a_{1}, \ldots, a_{n}\right)=\min \left(r_{a_{1}}+r_{a_{2}}+\cdots+r_{a_{n}}, 1\right) \cdot e^{j \psi_{2}\left(\theta_{1}, \theta_{2}, \ldots, \theta_{n}\right)}
$$

is a general complex-valued grouping function.

Now, we consider the way of constructing new complexvalued grouping functions from some given complex-valued grouping functions. In the case of real-valued grouping functions, it is possible to use some aggregation operations to obtain a new grouping function from some given grouping functions. Unfortunately, this approach fails for complex fuzzy arithmetic aggregation because it does not satisfy the property of amplitude monotonicity [35]. This means the weighted sum cannot be used to construct new complex-valued grouping functions.

Example 7. From Examples 2 and 3, $g(a, b)=\max (|a|,|b|)$. $e^{\theta_{a} \wedge \theta_{b}}$ and $f(a, b)=\max (|a|,|b|)$ are complex-valued general grouping functions. However, $h(a, b)=(g(a, b)+f(a, b) / 2)$ is not a complex-valued general grouping function since it does not satisfy $G C g_{n} 3$. We give a example such that $a_{1}<a_{2}$ but $\left|h\left(a_{1}, b\right)\right|>\left|h\left(a_{2}, b\right)\right|$; let $a_{1}=0.5 e^{2 \pi / 3}, \quad a_{2}=0.9 e^{\pi}$, and $b=0.5 e^{2 \pi / 3}$, then

$$
\begin{gathered}
\left|h\left(0.5 e^{2 \pi / 3}, 0.5 e^{2 \pi / 3}\right)\right|=\left|\frac{0.5 e^{2 \pi / 3}+0.5}{2}\right|=0.25, \\
\left|h\left(0.9 e^{\pi}, 0.5 e^{2 \pi / 3}\right)\right|=\left|\frac{0.9 e^{\pi}+0.5}{2}\right|=0.2 .
\end{gathered}
$$

Thus, $\left|h\left(0.5 e^{2 \pi / 3}, 0.5 e^{2 \pi / 3}\right)\right|>\left|h\left(0.9 e^{\pi}, 0.5 e^{2 \pi / 3}\right)\right|$.

However, it is possible to use product operation to obtain a new grouping function from some given grouping functions. The product of $\psi, \varphi: D^{n} \longrightarrow D$ is defined informally by

$$
(\psi \cdot \varphi)\left(a_{1}, \ldots, a_{n}\right)=\psi\left(a_{1}, \ldots, a_{n}\right) \times \varphi\left(a_{1}, \ldots, a_{n}\right) .
$$

Theorem 2. Let $\psi, \varphi$ be two n-dimensional general complexvalued grouping functions, then $t$ th power of $(\psi \cdot \varphi)$ also is an n-dimensional general complex-valued grouping function.

Proof. It is immediate that $\varphi$ is commutative and continuous. Now, we prove the properties $\left(G C g_{n} 1\right),\left(G C g_{n} 2\right)$, and $\left(G C g_{n} 3\right)$.

$\left(G C g_{n} 1\right):$ If $a_{i}=0$ for all $i=1,2, \ldots, n$, then $\psi\left(a_{1}, \ldots, a_{n}\right)=0$ and $\varphi\left(a_{1}, \ldots, a_{n}\right)=0$ since $\phi, \varphi$ are $n$-dimensional general complex-valued grouping functions. Then, $\psi^{t}\left(a_{1}, \ldots, a_{n}\right)=0 \ldots 0=0$.

$\left(G C g_{n} 2\right)$ : If there exists $i \in\{1,2, \ldots, n\}$ such that $a_{i}=1$, then $\phi\left(a_{1}, a_{2}, \ldots, a_{n}\right)=\varphi\left(a_{1}, a_{2}, \ldots, a_{n}\right)=1$ since $\phi, \varphi$ are n-dimensional general complex-valued grouping functions. Then, $\psi^{t}\left(a_{1}, \ldots, a_{n}\right)=1 \ldots 1=1$. $\left(G C g_{n} 3\right)$ : If $|a| \leq|b|$, then $\left|\psi\left(a, a_{2}, \ldots, a_{n}\right)\right| \leq \mid \psi\left(b, a_{2}\right.$ $\left.\ldots, a_{n}\right) \mid$ and $\left|\varphi\left(a, a_{2}, \ldots, a_{n}\right)\right| \leq\left|\varphi\left(b, a_{2} \ldots, a_{n}\right)\right|$ since $\phi, \varphi$ are n-dimensional general complex-valued grouping function. Then, $\mid \psi\left(a, a_{2}, \ldots, a_{n}\right) \times \varphi \quad\left(a, a_{2}\right.$, $\left.\ldots, a_{n}\right)|=| \psi \quad\left(a, a_{2}, \ldots, a_{n}\right)|\times| \varphi \quad\left(a, a_{2}, \ldots, a_{n}\right)|\leq| \psi$ 


$$
\begin{aligned}
& \left(b, a_{2}, \ldots, a_{n}\right)|\times| \varphi\left(b, a_{2}, \ldots, a_{n}\right)|=| \psi^{t}\left(b, a_{2}, \ldots, a_{n}\right) \times \varphi \\
& \left(b, a_{2}, \ldots, a_{n}\right) \mid .
\end{aligned}
$$

Similarly, we can get the following result.

Theorem 3. Let $\psi, \varphi$ be two $n$-dimensional general complexvalued 1-grouping functions, then their product $\psi \cdot \varphi$ also is an $n$-dimensional complex-valued 1-grouping function.

Proof. From Theorem 2 and the definition of $n$-dimensional complex-valued 1-grouping function, we only need to prove the following property:

$\left(C g_{n} 1\right):(\Rightarrow)$ If $(\psi \cdot \varphi)\left(a_{1}, \ldots, a_{n}\right)=\psi\left(a_{1}, \ldots, a_{n}\right)$ $\times \varphi\left(a_{1}, \ldots, a_{n}\right)=0$, then $\varphi\left(a_{1}, \ldots, a_{n}\right)=0$ or $\psi\left(a_{1}, \ldots, a_{n}\right)=0$. If $\psi\left(a_{1}, \ldots, a_{n}\right)=0$, then $a_{i}=0$ for all $i=1,2, \ldots, n$ since $\phi$ is an $n$-dimensional general complex-valued 1-grouping function. We have same result when $\varphi\left(a_{1}, \ldots, a_{n}\right)=0$.

Now, we consider the power of complex-valued grouping function. First, for positive integers $t$, the $t$ th power of $\psi: D^{n} \longrightarrow D$ is defined informally by

$$
\psi^{t}\left(a_{1}, \ldots, a_{n}\right)=\underbrace{\psi\left(a_{1}, \ldots, a_{n}\right) \times \cdots \times \psi\left(a_{1}, \ldots, a_{n}\right)}_{t \text { times }} .
$$

Theorem 4. Let $\psi$ be an n-dimensional general complexvalued grouping function, then $t$ th power of $\psi$ also is an $n$ dimensional general complex-valued grouping function.

Proof. It is immediate that $\varphi$ is commutative and continuous. Now, we prove the properties $\left(G C g_{n} 1\right),\left(G C g_{n} 2\right)$, and $\left(G C g_{n} 3\right)$.

$\left(G C g_{n} 1\right):$ If $a_{i}=0$ for all $i=1,2, \ldots, n$, then $\psi\left(a_{1}, \ldots, a_{n}\right)=0$ since $\phi$ is an $n$-dimensional general complex-valued grouping function. Then, $\psi^{t}\left(a_{1}, \ldots, a_{n}\right)=0 \ldots 0=0$.

$\left(G C g_{n} 2\right)$ : If there exists $i \in\{1,2, \ldots, n\}$ such that $a_{i}=1$, then $\phi\left(a_{1}, a_{2}, \ldots, a_{n}\right)=1$ since $\phi$ is an $n$-dimensional general complex-valued grouping function. Then, $\psi^{t}\left(a_{1}, \ldots, a_{n}\right)=1 \ldots 1=1$.

$\left(G C g_{n} 3\right):$ If $|a| \leq|b|$, then $\left|\psi\left(a, a_{2}, \ldots, a_{n}\right)\right|$ $\leq\left|\psi\left(b, a_{2} \ldots, a_{n}\right)\right|$ since $\phi$ is an $n$-dimensional general complex-valued grouping function. Then, $\left|\psi^{t}\left(a, a_{2}, \ldots, a_{n}\right)\right|=\left|\psi\left(a, a_{2}, \ldots, a_{n}\right)\right|^{t} \leq \mid \psi\left(b, a_{2}, \ldots\right.$, $\left.a_{n}\right)\left.\right|^{t}=\left|\psi^{t}\left(b, a_{2}, \ldots, a_{n}\right)\right|$.

Similarly, we can get the following result.

Theorem 5. Let $\psi$ be an n-dimensional general complexvalued 1-grouping function, then $t$ th power of $\psi$ also is an $n$ dimensional complex-valued 1-grouping function.

Proof. From Theorem 4 and the definition of $n$-dimensional complex-valued 1-grouping function, we only need to prove the following property:

$\left(C g_{n} 1\right):(\Rightarrow)$ If $\psi^{t}\left(a_{1}, \ldots, a_{n}\right)=\left(\psi\left(a_{1}, \ldots, a_{n}\right)\right)^{t}=0$, then $\psi\left(a_{1}, \ldots, a_{n}\right)=0$. Then, $a_{i}=0$ for all $i=1,2, \ldots, n$ since $\phi$ is an $n$-dimensional general complex-valued 1-grouping function

Remark 1. In the unit interval $[0,1], a \cdot b=1$ if and only if $a=b=1$. However, it is not true for the complex unit disk $D$ since $(-1) \cdot(-1)=1$. We cannot establish a theorem similar to Theorem 2 or Theorem 4 for $n$-dimensional complexvalued grouping (or 0 -grouping) function.

\section{Conclusions}

This paper introduced the concepts of complex-valued grouping, complex-valued 0-grouping, complex-valued 1grouping, and general complex-valued grouping functions. Then, we presented some construction methods for general complex-valued grouping functions. Our method includes the construction of a general real-valued grouping function and a continuous, commutative function $\psi:[0,2 \pi)^{n} \longrightarrow[0,2 \pi)$ satisfying the following property:

(i) There exists $i \in\{1,2, \ldots, n\}$ such that $\theta_{a_{i}}=0$ if and only if $\psi\left(\theta_{a_{1}}, \ldots, \theta_{a_{n}}\right)=0 \bmod 2 \pi$

Of course, the application and properties of complexvalued overlap/grouping functions need further investigation.

\section{Data Availability}

No data were used to support this study.

\section{Conflicts of Interest}

The authors declare that they have no conflicts of interest.

\section{Acknowledgments}

This research was funded by the National Science Foundation of China under Grant no. 62006168 and Zhejiang Provincial Natural Science Foundation of China under Grant no. LQ21A010001.

\section{References}

[1] H. Bustince, M. Pagola, R. Mesiar, E. Hlermeier, and F. Herrera, "Grouping, overlaps, and generalized bientropic functions for fuzzy modeling of pairwise comparisons," IEEE Transactions on Fuzzy Systems, vol. 20, pp. 405-415, 2012.

[2] G. Beliakov, A. Pradera, and T. Calvo, Aggregation Functions: A Guide for Practitioners, Springer, Berlin, Germany, 2007.

[3] A. Jurio, H. Bustince, M. Pagola, A. Pradera, and R. Yager, "Some properties of overlap and grouping functions and their application to image thresholding," Fuzzy Sets and Systems, vol. 229, pp. 69-90, 2013.

[4] M. Elkano, M. Galar, J. Sanz, and H. Bustince, "Fuzzy rule-based classification systems for multi-class problems using binary decomposition strategies: on the influence of $n$-dimensional overlap functions in the fuzzy reasoning method," Information Science, vol. 332, pp. 94-114, 2016.

[5] M. Elkano, M. Galar, J. Sanz et al., "Enhancing multi-class classification in FARC-HD fuzzy classifier: on the synergy between $\mathrm{n}$-dimensional overlap functions and decomposition 
strategies," IEEE Transactions on Fuzzy Systems, vol. 23, pp. 1562-1580, 2015.

[6] M. Elkano, M. Galar, J. A. Sanz et al., "Consensus via penalty functions for decision making in ensembles in fuzzy rulebased classification systems," Applied Soft Computing, vol. 67, 2017.

[7] H. Santos, L. Lima, B. Bedregal, G. P. Dimuro, M. Rocha, and H. Bustince, "Analyzing subdistributivity and superdistributivity on overlap and grouping functions," in Proceedings of the 8th International Summer School on Aggregation Operators (AGOP 2015), pp. 211-216, Katowice, Poland, July 2015.

[8] H. Santos, G. P. Dimuro, T. C. Asmus et al., "General grouping functions," in Proceedings of 18th International Conference on Information Processing and Management of Uncertainty in Knowledge-Based Systems, ser. Communications in Computer and Information Science, Springer, Cham, Basel, Switzerland, June 2020.

[9] T. C. Asmus, G. P. Dimuro, B. Bedregal, J. A. Sanz, S. Pereira, and H. Bustince, "General interval-valued overlap functions and interval-valued overlap indices," Information Science, vol. 527 , pp. $27-50,2020$.

[10] B. Bedregal, H. Bustince, E. Palmeira, G. Dimuro, and J. Fernandez, "Generalized interval-valued OWA operators with interval weights derived from interval-valued overlap functions," International Journal of Approximate Reasoning, vol. 90, pp. 1-16, 2017.

[11] B. Bedregal, G. P. Dimuro, H. Bustince, and E. Barrenechea, "New results on overlap and grouping functions," Information Science, vol. 249, pp. 148-170, 2013.

[12] D. Gómez, J. T. Rodríguez, J. Montero, H. Bustince, and E. Barrenechea, "N-dimensional overlap functions," Fuzzy Sets and Systems, vol. 287, pp. 57-75, 2016.

[13] J. Qiao and B. Q. Hu, "On the migrativity of uninorms and nullnorms over overlap and grouping functions," Fuzzy Sets and Systems, vol. 346, pp. 1-54, 2017.

[14] J. Qiao and B. Q. Hu, "On the distributive laws of fuzzy implication functions over additively generated overlap and grouping functions," IEEE Transactions on Fuzzy Systems, vol. 26, no. 4, pp. 2421-2433, 2017.

[15] J. Qiao and B. Q. Hu, "On multiplicative generators of overlap and grouping functions," Fuzzy Sets and Systems, vol. 332, pp. 1-24, 2018.

[16] J. Qiao and B. Q. Hu, "On interval additive generators of interval overlap functions and interval grouping functions," Fuzzy Sets and Systems, vol. 323, pp. 19-55, 2017.

[17] G. P. Dimuro, B. Bedregal, and R. H. N. Santiago, "On (G, N)implications derived from grouping functions," Information Science, vol. 279, pp. 1-17, 2014.

[18] G. P. Dimuro, B. Bedregal, H. Bustince, A. Jurio, M. Baczynski, and K. Mis, "QL operations and QL-implication functions constructed from tuples $(\mathrm{O}, \mathrm{G}, \mathrm{N})$ and the generation of fuzzy subsethood and entropy measures," International Journal of Approximate Reasoning, vol. 82, pp. 170-192, 2017.

[19] D. Ramot, R. Milo, M. Friedman, and A. Kandel, "Complex fuzzy sets," IEEE Transactions on Fuzzy Systems, vol. 10, pp. 171-186, 2002.

[20] D. Ramot, M. Friedman, G. Langholz, and A. Kandel, "Complex fuzzy logic," IEEE Transactions on Fuzzy Systems, vol. 11, pp. 450-461, 2003.

[21] B. Hu, L. Bi, and S. Dai, "The orthogonality between complex fuzzy sets and its application to signal detection," Symmetry, vol. 9, p. 175, 2017.
[22] X. Ma, J. Zhan, M. Khan, M. Zeeshan, S. Anis, and A. S. Awan, "Complex fuzzy sets with applications in signals," Computational and Applied Mathematics, vol. 38, no. 4, p. 150, 2019.

[23] Z. Chen, S. Aghakhani, J. Man, and S. Dick, "ANCFIS: a neuro-fuzzy architecture employing complex fuzzy sets," IEEE Transactions on Fuzzy Systems, vol. 19, pp. 305-322, 2011.

[24] C. Li, "Complex neuro-fuzzy ARIMA forecasting. a new approach using complex fuzzy sets," IEEE Transactions on Fuzzy Systems, vol. 21, pp. 567-584, 2013.

[25] J. Ma, G. Zhang, and J. Lu, "A method for multiple periodic factor prediction problems using complex fuzzy sets," IEEE Transactions on Fuzzy Systems, vol. 20, pp. 32-45, 2012.

[26] H. Garg and D. Rani, "Some generalized complex intuitionistic fuzzy aggregation operators and their application to multicriteria decision-making process," Arabian Journal for Science and Engineering, vol. 44, pp. 2679-2698, 2018.

[27] D. Rani and H. Garg, "Complex intuitionistic fuzzy power aggregation operators and their applications in multicriteria decision-making," Expert Systems, vol. 35, no. 6, Article ID e12325, 2018.

[28] S. Dai, L. Bi, and B. Hu"Distance measures between the interval-valued complex fuzzy sets," Mathematics, vol. 7, p. 549, 2019.

[29] S. Dai, "Complex fuzzy ordered weighted distance measures," Iranian Journal of Fuzzy System, vol. 17, no. 6, pp. 107-114, 2020.

[30] B. Hu, L. Bi, S. Dai, and S. Li, "Distances of complex fuzzy sets and continuity of complex fuzzy operations," Journal of Intelligent and Fuzzy Systems, vol. 35, pp. 2247-2255, 2018.

[31] S. Dai, "A generalization of rotational invariance for complex fuzzy operations," IEEE Transactions on Fuzzy Systems, vol. 29, pp. 1152-1159, 2021.

[32] S. Dai, "On partial orders in complex fuzzy logic," IEEE Transactions on Fuzzy Systems, vol. 29, pp. 698-701, 2021.

[33] S. Dick, “Towards complex fuzzy logic," IEEE Transactions on Fuzzy Systems, vol. 13, pp. 405-414, 2005.

[34] Y. Chen, L. Bi, B. Hu, and S. Dai, "General complex-valued overlap functions," Journal of Mathematics, vol. 2021, Article ID 6613730, 6 pages, 2021.

[35] L. Bi, S. Dai, B. Hu, and S. Li, "Complex fuzzy arithmetic aggregation operators," Journal of Intelligent and Fuzzy Systems, vol. 36, pp. 2765-2771, 2019. 